\title{
PDCD5 expression predicts a favorable outcome in patients with hepatocellular carcinoma
}

\author{
DA-ZHI FU, YING CHENG, HUI HE, HAI-YANG LIU and YONG-FENG LIU
}

Department of General Surgery, First Affiliated Hospital of China Medical University, Shenyang 110001, Liaoning, P.R. China

Received April 17, 2013; Accepted June 10, 2013

DOI: 10.3892/ijo.2013.1993

\begin{abstract}
Liver cancer in men is the fifth most frequently diagnosed cancer worldwide. Programmed cell death 5 (PDCD5) is an apoptosis related gene and plays an important role in the pathogenesis and development of cancer. In this study, we confirmed that the levels of PDCD5 mRNA and protein were lower in hepatocellular carcinoma (HCC) tissue compared to normal tissue. PDCD5 expression was significantly associated with HBV infection, tumor number, lymph node metastasis and the survival time of the patients with HCC. In addition, the serum levels of PDCD5 and AFP in the patients were significantly positively correlated. We also confirmed that upregulation of PDCD5 was able to inhibit cell proliferation and mobility, induce apoptosis and $\mathrm{G}_{1}$ arrest. Interestingly, PDCD5 also inhibited proteasome activity similarly to the proteosome inhibitor MG132. PDCD5 could be considered as a reliable marker of favorable prognosis of $\mathrm{HCC}$ patients.
\end{abstract}

\section{Introduction}

Liver cancer in men is the fifth most frequently diagnosed cancer worldwide but the second most frequent cause of cancer death. An estimated 748,300 new liver cancer cases and 695,900 cancer deaths occurred worldwide in 2008 (1). Half of these cases and deaths were estimated to occur in China (2). Among primary liver cancers, hepatocellular carcinoma (HCC) represents the major histological subtype, accounting for $70-85 \%$ of the total liver cancer burden worldwide (3).

TF-1 apoptosis-related gene 19 (TFAR19) was first cloned as an upregulated gene from TF-1 cells undergoing apoptosis following serum withdrawn by Liu et al (4) in the Peking University Center for Human Disease Genomics in 1999, then designated as programmed cell death 5 (PDCD5)

Correspondence to: Dr Yong-Feng Liu, Department of General Surgery, First Affiliated Hospital of China Medical University, 155 Nanjing Road, Heping District, 110001 Shenyang, Liaoning, P.R. China

E-mail: yfliu_2006@163.com

Key words: hepatocellular carcinoma, PDCD5, apoptosis, cell cycle, proteasome by International Human Gene Nomination Committee (GenBank accession no. AF014955). PDCD5 gene is located on chromosome 19q12-q13.1 (5) and the integrated PDCD5 protein contains 125 amino acid (aa) residues (6). Decreased expression of PDCD5 has been characterized for human tumors including breast cancer (7), gastric cancer (8), astrocytic glioma (9) and chronic myelogenous leukemia (10). The scientists in the Peking University Center for Human Disease Genomics established an ELISA method for detecting soluble PDCD5 protein (11). Wang et al (12) illustrated a significant elevation of PDCD5 concentrations in both plasma and synovial fluid of knee rheumatoid arthritis (RA) patients compared to osteoarthritis (OA) patients and the control plasma concentrations. The serum PDCD5 levels in breast cancer, gastrointestinal tract cancer and lung cancer patients were separately compared with healthy persons by Wang et al (13). However, the significance of serum PDCD5 and its changes in various cancers await further investigations.

Xu et al (14) demonstrated that PDCD5 interacts with Tip60, enhances the histone acetylation and p53 K120 acetylation and promotes the expression of Bax, consequently accelerating apoptosis. In their recent study, novel evidence demonstrated that PDCD5 is a p53 regulator during gene expression and cell cycle (15). Li et al (16) found that PDCD5 promotes cisplatininduced apoptosis of glioma cells via activating mitochondrial apoptotic pathway. Han et al (17) confirmed that PDCD5 shows a greater inhibitory effect on the Ras/Raf/MEK/ERK signaling pathway in the human osteosarcoma cell line MG-63. However, the molecular mechanisms underlying PDCD5 functions during cell growth, proliferation and apoptosis remain largely unclear.

In this study, we first demonstrate that PDCD5 expression is correlated with clinicopathological features and patient survival and it may be a useful predictor of prognosis in patients with HCC after surgical resection. Second, we compared the PDCD5 levels in peripheral blood serum from cancer patients with matched normal volunteers. The susceptibility of HCC cells to human PDCD5 was also investigated in vitro.

\section{Materials and methods}

Cell culture and tumor specimens. Human liver cancer cell lines, HepG2 and Hep3B, were cultured in DMEM (Hyclone, Logan, UT, USA) containing $10 \%$ fetal bovine serum (Invitrogen Gibco, Carlsbad, CA, USA) and incubated in 
a $5 \% \mathrm{CO}_{2}$ incubator at $37^{\circ} \mathrm{C}$. Liver cancer tissue specimens and their adjacent normal liver tissues were derived from 56 patients undergoing surgical resection of primary hepatocellular carcinoma without prior chemotherapeutic treatment or radiotherapy. The adjacent non-malignant tissue was $\geq 1$-cm distance from the tumor margin. Resections were performed at Department of General Surgery, First Affiliated Hospital of China Medical University from January 2008 to December 2012. All patients approved the use of tumor tissues for clinical research and China Medical University Ethics Committee approved the research protocols. Basic patient information is summarized in Table I.

Quantitative real-time PCR. Total RNA was isolated using an RNeasy Mini kit (Biomed, Beijing, China). First strand cDNA was reverse transcribed with $1 \mu \mathrm{g}$ of total RNA, using Takara Reverse Transcription kit (Takara, Dalian, China) and oligo(dT) 15 primers (Takara). The resultant cDNA was then used for quantitative $P C R$ reactions. The $P D C D 5$ primers were: sense: 5'-CTGAGGAGACAGAGGCTGGC-3' and antisense: 5'-TTTCTGCTTCCCTGTGCTTTG-3'. The housekeeping genes, GAPDH and $\beta$-actin, were used as internal controls for normalization of the results. The $G A P D H$ primers were: sense: 5'-AGAAGGCTGGGGCTCATTTG-3' and antisense: 5'-AGGGGCCATCCACAGTCTTC-3'. The $\beta$-actin primers were: sense: 5'-CTCCCTGGAGAAGAGCTACGA-3' and antisense: 5'-GTGGGACTTCCAGAACTGCA-3'. Amplification of PDCD5, GADPH and $\beta$-actin was performed with 1 cycle at $95^{\circ} \mathrm{C}$ for $10 \mathrm{~min}$ and 40 cycles of $95^{\circ} \mathrm{C}$ for $15 \mathrm{sec}$ and $60^{\circ} \mathrm{C}$ for $60 \mathrm{sec}$. Calculation of the relative expression of each transcript was performed using the $2^{-\Delta \Delta C t}$ method (18).

$R N A$ isolation and reverse transcriptase-polymerase chain reaction (RT-PCR). Total RNA was isolated from HepG2 and Hep3B cells using the RNeasy Mini kit (Biomed, Beijing, China). First strand cDNA was reverse transcribed with $1 \mu \mathrm{g}$ total RNA, using the Takara Reverse Transcription kit (Takara) and oligo(dT)-15 primers (Takara) according to the manufacturer's instructions. The primers of $P D C D 5$ and $G A P D H$ were used as described above. PCR amplification of cDNA was performed in $20 \mu \mathrm{l}$ mixtures. Finally, products were resolved by $1.5 \%$ agarose gel electrophoresis and visualized by ethidium bromide staining and a UV imaging system (UVP, LLC, Upland, CA, USA).

Immunohistochemical staining (IHC). IHC of $4-\mu \mathrm{m}$ sections of paraffin-embedded specimens was performed using the antiPDCD5 polyclonal antibody (Beijing Biosea Biotechnology, Beijing, China). Briefly, after deparaffinization and hydration, the endogenous peroxidase activity was quenched by a $30-\mathrm{min}$ incubation in a mixture of $0.3 \%$ hydrogen peroxide solution in $100 \%$ methanol. The sections were blocked for $2 \mathrm{~h}$ at room temperature with $1.5 \%$ blocking serum in PBS and incubated with anti-PDCD5 antibody (1:200 dilution) at $4^{\circ} \mathrm{C}$ in a moist chamber overnight, followed by incubation with Envision reagent (Dako, Carpinteria, CA, USA) and color development in 3,3'-diaminobenzidine tetrahydrochloride (DAB, SigmaAldrich, Carlsbad, CA, USA). The slides were then lightly counterstained with hematoxylin, dehydrated with ethanol, cleaned with xylene and mounted. Adjacent non-cancer tissues were used as controls. Sections treated without primary antibodies were used as negative controls. The positive percentage of counted cells was graded semi-quantitatively according to a four-tier scoring system: negative (-), 0-5\%; weakly positive (+), 6-25\%; moderately positive (++), 26-50\%; and strongly positive (+++), 51-100\%.

Preparation of blood samples and measurement of PDCD5 and AFP in serum. Peripheral blood was obtained from 58 patients with $\mathrm{HCC}$ and 58 healthy volunteers. Samples were clotted for $30 \mathrm{~min}$ and then centrifuged for $10 \mathrm{~min}$ at $1000 \mathrm{x} \mathrm{g}$. All samples were then frozen and stored at $-80^{\circ} \mathrm{C}$ until required for assay. Concentration of PDCD5 in serum was assayed using enzyme-linked immunosorbent assay (ELISA) kit for PDCD5 (USCN Life Science Inc., Houston, TX, USA). Concentration of AFP in serum was measured using an ELISA kit from Diagnostic Automation/Cortez Diagnostics Inc (Calabasas, CA, USA).

Plasmid and transfection. The plasmid, pcDNA3.1-PDCD5, was kindly provided by Mr. Xiao-Rui Han (China Medical University, Shenyang). Transfection of the plasmid into HepG2 cells or Hep3B was performed using Lipofectamine ${ }^{\mathrm{TM}} 2000$ (Invitrogen) according to the manufacturer's instructions. Cell lines after transfection were named HepG2-T and Hep3B-T. Cell lines transfected with pcDNA3.1 was used as a mock and named HepG2-M and Hep3B-M.

Immunofluorescence. Transfected cells were washed with PBS, fixed in $4 \%$ paraformaldehyde, permeabilized in $1 \%$ Triton X-100 for 5 min and blocked with 5\% bovine serum albumin in PBS containing 0.5\% Triton X-100 for $1 \mathrm{~h}$. PDCD5 expression was detected using anti-PDCD5 (Beijing Biosea Biotechnology) antibody for $1 \mathrm{~h}$ at room temperature. Cells were washed with PBS and incubated with appropriate secondary fluorophore-conjugated antibody for $1 \mathrm{~h}$ at room temperature, washed with PBS and mounted using SlowFade ${ }^{\circledR}$ Gold Antifade reagent (Invitrogen). Secondary antibody used for detection of PDCD5 was Alexa Fluor ${ }^{\circledR} 594$ Donkey AntiGoat $\operatorname{IgG}(\mathrm{H}+\mathrm{L})$ (Invitrogen).

Evaluation of cell viability by MTT assay. Cell viability was assayed using 3-(4,5-dimethylthiazolyl)-2,5-diphenyltetrazoliumbromide (MTT) assays (Sigma). Briefly, cells were plated in 96-well plates (1,500 cells per well). After $24 \mathrm{~h}, 0.5 \mathrm{mg} / \mathrm{ml}$ MTT was added to each well. Four hours later, cells were lysed with dimethyl sulfoxide (DMSO) and absorbance rates were measured at 550-560 nm using a microplate reader (Bio-Rad, Hercules, CA, USA). Proteasome inhibitor, MG132, purchased from Calbiochem (La Jolla, CA, USA), was used as a control in the following experiments.

TUNEL assay. For apoptosis detection, HepG2 cells, HepG2-M cells, HepG2-T cells, HepG2 treated with MG132, Hep3B cells, Hep3B-M cells, Hep3B-T cells and Hep3B cells treated with MG132 were washed in PBS, fixed, permeabilized and subjected to TUNEL labeling using an In Situ Cell Death Detection kit (Keygen, Nanjing, China) according to the manufacturer's protocol. After counterstaining with DAPI $(1 \mu \mathrm{g} / \mathrm{ml})$, photographic images were taken using an 
Table I. PDCD5 expression associated with demographic and biological parameters in 56 hepatocellular carcinoma samples.

\begin{tabular}{|c|c|c|c|c|c|c|c|c|}
\hline \multirow[b]{2}{*}{ Clinicopathological features } & \multirow[b]{2}{*}{$\mathrm{n}$} & \multicolumn{7}{|c|}{ PDCD5 expression } \\
\hline & & - & + & ++ & +++ & PR $(\%)$ & $\chi^{2}$ & P-value \\
\hline Gender & & & & & & & 2.57 & 0.462 \\
\hline Female & 20 & 12 & 4 & 1 & 3 & 40.0 & & \\
\hline Male & 36 & 28 & 4 & 2 & 2 & 22.2 & & \\
\hline Age (years) & & & & & & & 4.87 & 0.181 \\
\hline$<55$ & 26 & 15 & 5 & 2 & 4 & 42.3 & & \\
\hline$\geq 55$ & 30 & 25 & 3 & 1 & 1 & 16.7 & & \\
\hline Tumor number & & & & & & & 8.89 & 0.030 \\
\hline Multiple & 38 & 27 & 6 & 0 & 5 & 28.9 & & \\
\hline Solitary & 18 & 13 & 2 & 3 & 0 & 27.8 & & \\
\hline Differentiation & & & & & & & 2.06 & 0.559 \\
\hline Differentiated & 27 & 20 & 4 & 2 & 1 & 25.9 & & \\
\hline Undifferentiated & 29 & 20 & 4 & 1 & 4 & 31.0 & & \\
\hline Portal invasion & & & & & & & 3.14 & 0.370 \\
\hline- & 17 & 14 & 1 & 0 & 2 & 17.6 & & \\
\hline+ & 39 & 26 & 7 & 3 & 3 & 33.3 & & \\
\hline Lymph node metastasis & & & & & & & 11.07 & 0.011 \\
\hline- & 22 & 11 & 7 & 2 & 2 & 50.0 & & \\
\hline+ & 34 & 29 & 1 & 1 & 3 & 14.7 & & \\
\hline Tumor size $(\mathrm{cm})$ & & & & & & & 5.62 & 0.131 \\
\hline$<5$ & 25 & 15 & 5 & 3 & 2 & 40.0 & & \\
\hline$\geq 5$ & 31 & 25 & 3 & 0 & 3 & 19.4 & & \\
\hline HBV infection & & & & & & & 8.77 & 0.030 \\
\hline- & 18 & 10 & 4 & 0 & 4 & 44.4 & & \\
\hline+ & 38 & 30 & 4 & 3 & 1 & 21.1 & & \\
\hline
\end{tabular}

PR, positive rate; $\chi^{2}$ value, $\chi^{2}$ distribution.

Olympus CX71 fluorescence microscope (Olympus, Tokyo, Japan). TUNEL-positive nuclei were stained green and all other nuclei were stained blue (19).

Cell apoptosis assay. Cells $\left(5 \times 10^{5}\right)$ were collected without EDTA and washed with PBS. A $500-\mu 1$ binding buffer, $5 \mu 1$ Annexin V-FITC and $5 \mu \mathrm{l}$ propidium iodide (PI) were added into the suspension in this order and mixed at room temperature in dark for $10 \mathrm{~min}$. The examination was performed by flow cytometry within $1 \mathrm{~h}$.

Cell cycle assay. Cells seeded on 6-well plates were treated and then collected. After being washed with PBS three times, the cell suspension was fixed with $70 \%$ ethanol, incubated with RNAse $\mathrm{A}$ at $37^{\circ} \mathrm{C}$. After staining with $400 \mu \mathrm{l} \mathrm{PI}$, the suspension was evaluated by flow cytometry.

In vitro wound healing assay. Cells were grown in a 6-well dish. A confluent monolayer of cells was scratched with a $200-\mu 1$ pipette tip to create a wound. Cells were washed twice with PBS and then supplemented with medium and incubated for $4 \mathrm{~h}$ at $37^{\circ} \mathrm{C}$. Cell migration into the wounded area was monitored microscopically. Images were captured at the interface of the unwounded and wounded areas.

265 proteasome activity assay. $26 \mathrm{~S}$ proteasome function was assayed as described previously (20). The assay is based on detection of the fluorophore 7-amino-4-methylcoumarin (AMC) after cleavage from the labeled substrate Suc-LLVY-AMC (Boston Biochem, Boston, MA, USA). These fluorogenic proteasome substrates were added to the cell lysate at a final concentration of $80 \mu \mathrm{M}$ in $1 \%$ DMSO. ATP-dependent cleavage activity was monitored continuously by detection of free 7-amido-4-methylcoumarin using a microplate reader (Bio-Rad) at $380 / 460 \mathrm{~nm}$, at $37^{\circ} \mathrm{C}$.

Western blot analysis. The total protein was extracted from human liver cancer cell lines and liver tissues using RIPA buffer (Sigma). The protein concentration was determined with BCA Protein Assay kit (Pierce, Rockford, IL, USA). 

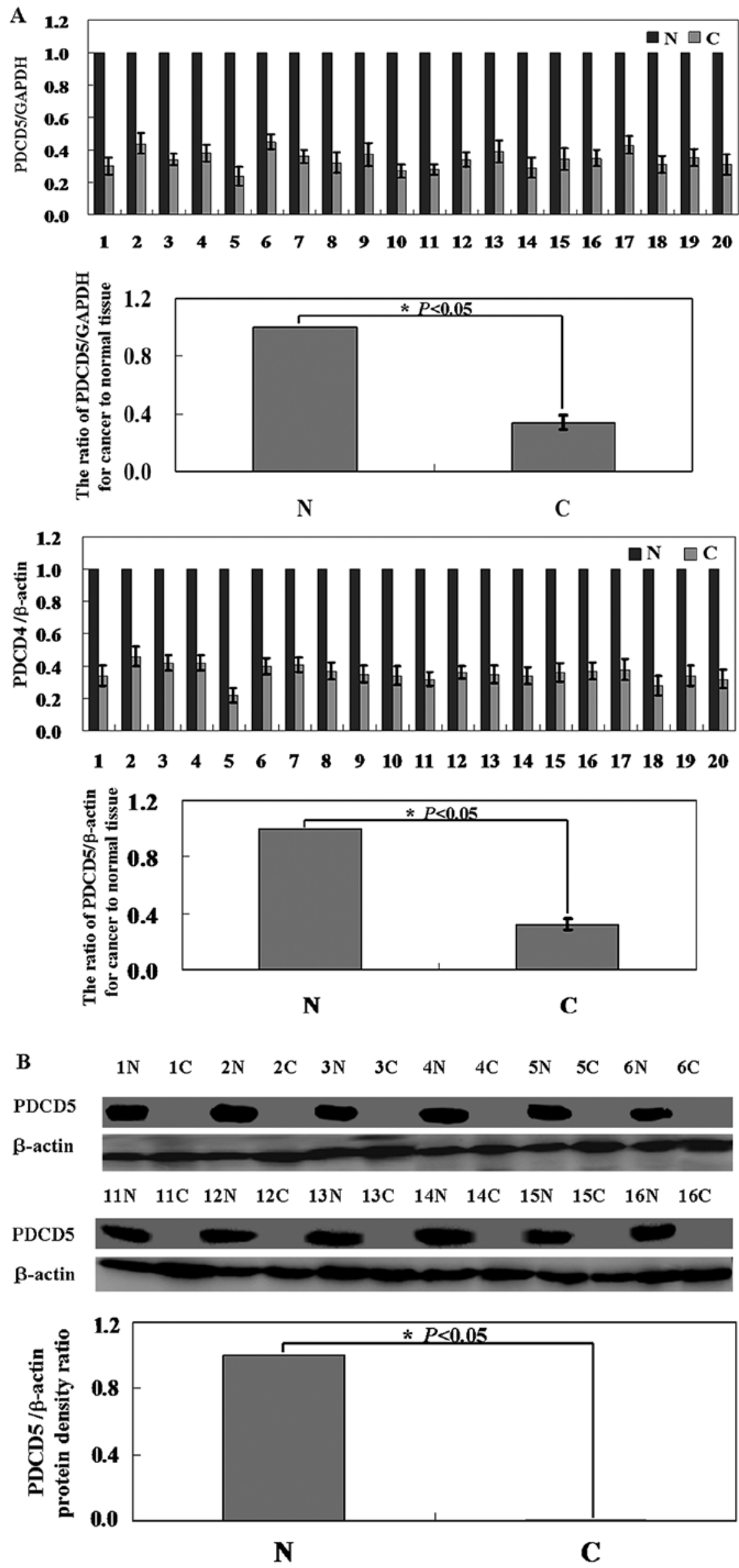

Figure 1. (A) The level of PDCD5 mRNA was measured in specimens by using real-time PCR. GADPH and $\beta$-actin were used as internal controls. (B) Representative results of two pairs of HCC and corresponding normal tissue by western blotting. $\beta$-actin was used as an internal control. 
C

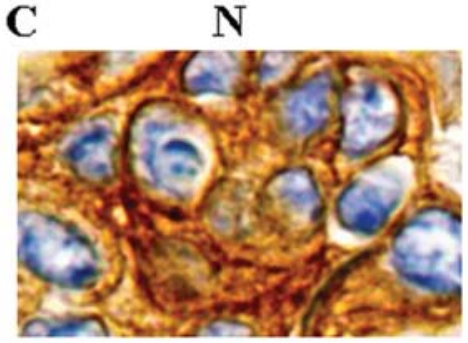

C

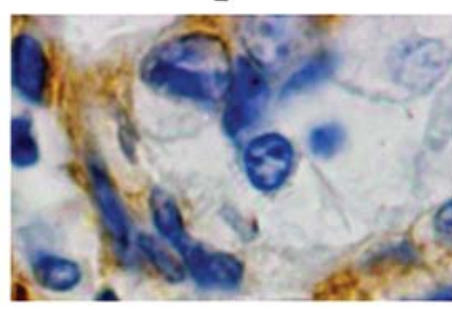

NC

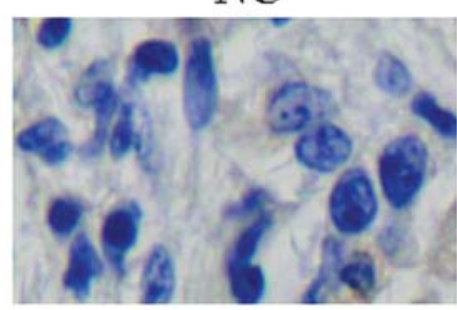

Figure 1. Continued. (C) Immunohistochemical staining for PDCD5 protein in specimens. PDCD5 was stained yellow with granules and localized to the cytoplasm. The nuclei were counterstained with hematoxylin. N, normal; C, cancer; NC, negative control.

For performing nitrocellulose membrane blotting, $60 \mu \mathrm{g}$ cell lysates were separated on a $10 \%$ SDS-PAGE. The blotted membranes were blocked with 5\% BSA in TBST for $2 \mathrm{~h}$ at room temperature and incubated with each primary antibody overnight. The primary antibodies were PDCD5 (Abcam, Cambridge, UK) and $\beta$-actin (Santa Cruz Biotechnology, Santa Cruz, CA, USA). The reaction was followed by probing with peroxidase-coupled secondary antibodies at dilutions ranging from 1:1,000 to 1:2,000 (Amersham Biosciences, Needham, MA, USA) and binding results were visualized by enhanced chemiluminescence (Amersham Pharmacia, Piscataway, NJ, USA).

Statistical analysis. Statistical analyses were performed using SPSS 15.0 software (SPSS, Chicago, IL, USA). Comparisons were made using $\chi^{2}$ tests, the Wilcoxon signed-rank test and the t-test. Overall survival was analyzed using the KaplanMeier method and the significance of differences in survival rates was estimated using the log-rank test. Values of $\mathrm{P}<0.05$ were considered significant.

\section{Results}

PDCD5 is downregulated in HCC tissues compared with normal tissues. To initially confirm the roles of PDCD5 expression in human liver cancer development and progression, we assayed the expression of PDCD5 in 56 pairs of human hepatocellular carcinomas and corresponding adjacent normal tissues. Levels of PDCD5 mRNA and protein in the hepatoma tissue were significantly lower than those in corresponding adjacent non-cancer tissue (Fig. $1 \mathrm{~A}$ and $\mathrm{B} ; \mathrm{P}<0.05$ ). In Fig. $1 \mathrm{C}$, the results of immunohistochemical staining showed that positive staining was seen in the cytoplasm of the normal cells, in contrast, almost no positive cells were seen in cancer tissue. Moreover, the PDCD5 expression was correlated statistically with HBV infection $(\mathrm{P}=0.030)$, tumor number $(\mathrm{P}=0.030)$ and lymph node metastasis $(\mathrm{P}=0.011)$. There was no significant correlation between PDCD5 expression and age, sex, tumor number, tumor size, differentiation and portal invasion (Table I, P>0.05). HCC patients with PDCD5 expression were associated with a significantly better survival rate than those without PDCD5 expression (Fig. 2, P=0.038).

The serum levels of PDCD5 and AFP detection in patients with HCC. The serum PDCD5 contents in $58 \mathrm{HCC}$ patients ranged from $4.41 \mathrm{pg} / \mathrm{ml}$ to $1.72 \mathrm{ng} / \mathrm{ml}$ with a median of $421.83 \pm 12.52 \mathrm{pg} / \mathrm{ml}$. There was statistical significance

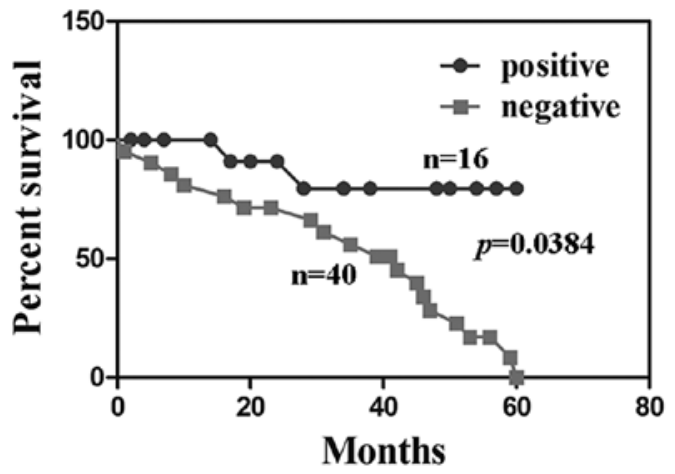

Figure 2. Kaplan-Meier curves for cumulative survival rate of the patients with HCC according to the PDCD5 expression.

compared with the serum PDCD5 level in healthy persons (Fig. 3A, P<0.05). Serum AFP levels in the HCC patients $(6.24 \mathrm{pg} / \mathrm{ml}$ to $1.53 \mathrm{ng} / \mathrm{ml})$ were significantly higher than that in the control group $(32.67 \mathrm{pg} / \mathrm{ml}$ to $2.46 \mathrm{ng} / \mathrm{ml}$ ) (Fig. 3B, $\mathrm{P}<0.05)$. The serum levels of PDCD5 and AFP in $58 \mathrm{HCC}$ patients were significantly positively correlated $(\mathrm{r}=0.913)$ (Fig. 3C, $\mathrm{P}<0.05$ ).

Establishment of PDCD5-overexpressing HepG2 and Hep3B cells. To investigate further the phenomenon and mechanism of the PDCD5-mediated regulation of liver cancer cell growth and metastasis, cell lines, HepG2 and Hep3B, were selected for PDCD5 gene enhancement. After pcDNA3.1-PDCD5 was transfected into the two hepatoma cell lines, PDCD5 mRNA and protein levels were significantly increased compared to untransfected cells by using RT-PCR and western blotting, respectively (Fig. 4A and B). Immunofluorescence analysis showed PDCD5 was localized in the cytoplasm of transfected cells (Fig. 4C). These results collectively suggested that the transfection was successful.

PDCD5 displays antitumor activity in liver cancer cells. Since PDCD5 mRNA and protein levels are decreased in liver cancer tissues and cells, we determined whether its exogenous expression would reduce tumorigenicity of liver cancer cells. The MTT assay showed that the proliferation rate of PDCD5-expressing cells was decreased compared to untransfected and mock-transfected cells (Fig. 5A, $\mathrm{P}<0.05$ ). TUNEL assay confirmed that the number of apoptotic cells in the transfected group was significantly more than 

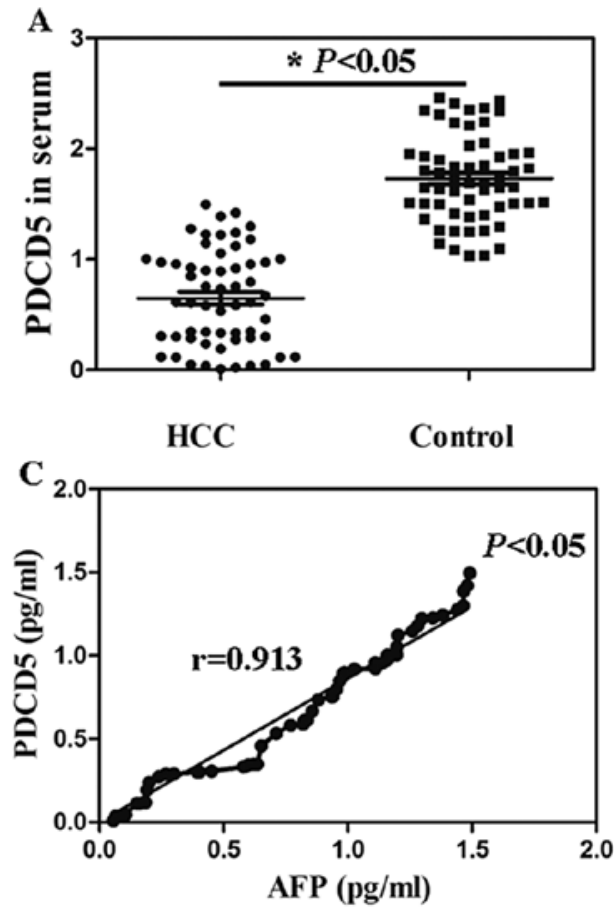

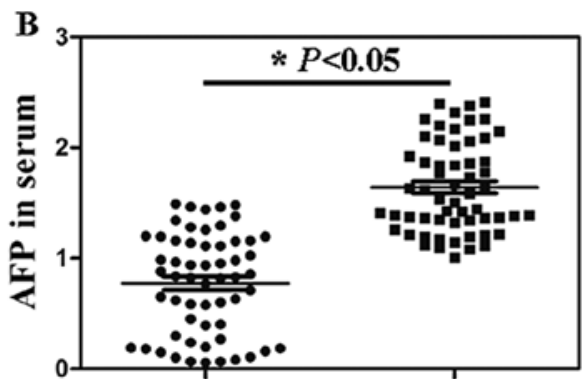

HCC

Figure 3. PDCD5 and AFP in serum of HCC patients and healthy controls. (A) Serum PDCD5 levels from patients with $\mathrm{HCC}(\mathrm{n}=58)$ vs healthy controls $(\mathrm{n}=58)$. (B) Serum AFP levels from patients with HCC $(\mathrm{n}=58)$ vs healthy controls $(\mathrm{n}=58)$. (C) Positive correlation between serum levels of PDCD5 and AFP in $\mathrm{HCC}$ patients $(\mathrm{n}=58)$.

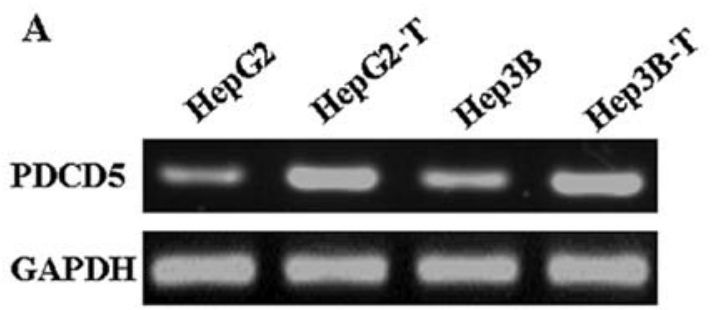

C HepG2

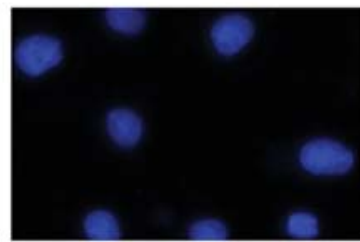

HepG2-T

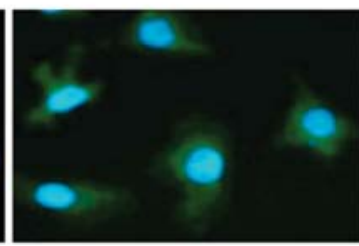

B

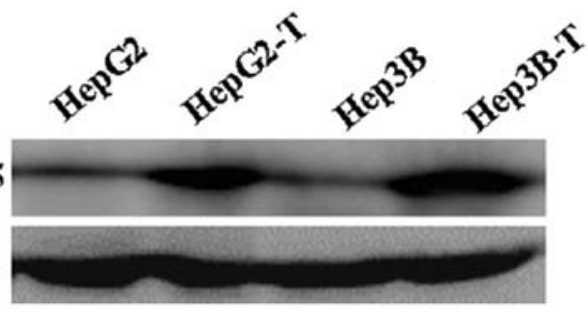

Hep3B

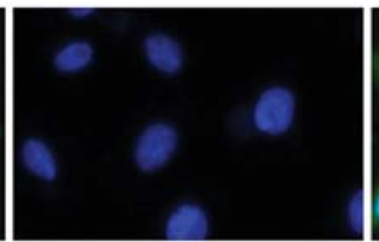

Hep3B-T

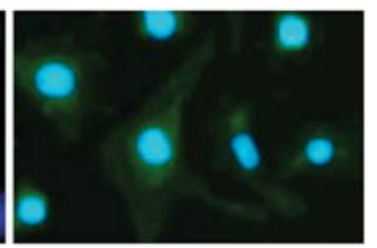

Figure 4. The levels of PDCD5 protein and mRNA were measured in HCC cell lines. (A) The mRNA level of PDCD5 was determined by RT-PCR. GAPDH was used as an internal control. (B) The expression of PDCD5 protein was determined by western blotting of HepG2 cells and Hep3B cells and the two cells transfected with pcDNA3.1-PDCD5. $\beta$-actin expression was used as an internal control. (C) Detection of PDCD5 in transfected and untransfected cells by immunofluorescence. PDCD5 protein was located predominantly in the cytoplasm.

untransfected group and mock-transfected group (Fig. 5B). To quantify apoptotic cells, Annexin V-FITC and PI double staining was performed. In cells expressing PDCD5, the apoptotic ratio was 3-6 times higher than that of untransfected and mock-transfected cells. We determined that the percentage of apoptosis in PDCD5-expressing HepG2 cells was 3.86\%, significantly higher compared to untransfected $(0.73 \%)$ and mock-transfected (1.12\%) HepG2 cells (Fig. 5C, $\mathrm{P}<0.05$ ). The apoptotic percentage of PDCD5-expressing Hep3B cells was $3.75 \%$ compared to untransfected $(1.23 \%)$ and mocktransfected (1.34\%) Hep3B cells (Fig. 5C, P<0.05). PI staining of cells revealed that PDCD5-expressing cells were arrested in $\mathrm{G}_{1}$ phase (Fig. 5D, $\mathrm{P}<0.05$ ). We determined the mobility change in PDCD5-expressing cells using the wound healing assay. The percentage of wound closure of PDCD5-positive cells was decreased when compared to untransfected and mock-transfected cells (Fig. 5E, $\mathrm{P}<0.05$ ).

PDCD5 inhibits proteasome activity in liver cancer cells. In order to determine whether PDCD5 could impact on the proteasome, we compared the response of MG132 treated liver cancer cells with PDCD5 treated cells. The proteasome activity of both PDCD5-expressing HepG2 cells and PDCD5expressing Hep3B cells was lower than that of untransfected and mock-transfected cells (Fig. 5F, P<0.05). HepG2 cells and Hep3B cells after MG132 treatment also showed increased apoptosis, $G_{1}$ arrest and decreased mobility. Thus, the results showed that MG132 has the same effect as PDCD5 in liver 


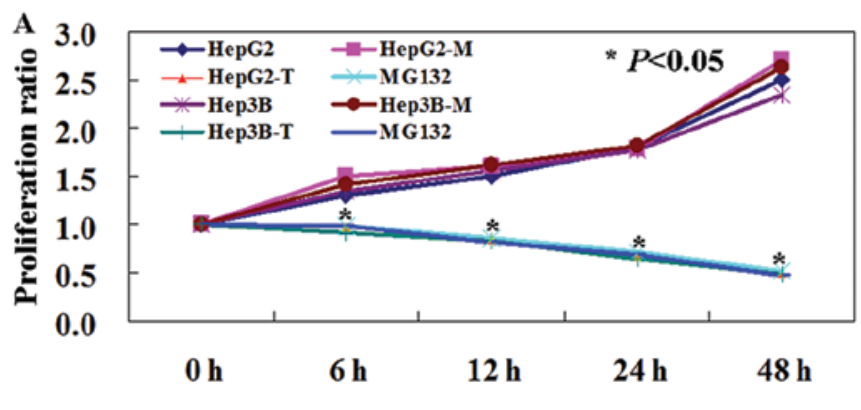

Figure 5. The antitumor roles of PDCD5 protein in HCC cells. (A) MTT assays were performed to determine the proliferation ratio of HCC cells with PDCD5 transfection compared with untransfected ones. (B) Representative TUNEL staining images of transfected cells and untransfected ones. Apoptotic cells with DNA fragmentation were stained positively as green nuclei. (C) The proportion of apoptotic cells (early apoptosis) was determined by double-staining with Annexin V/FITC and PI.

B

TUNEL

DAPI

Merge
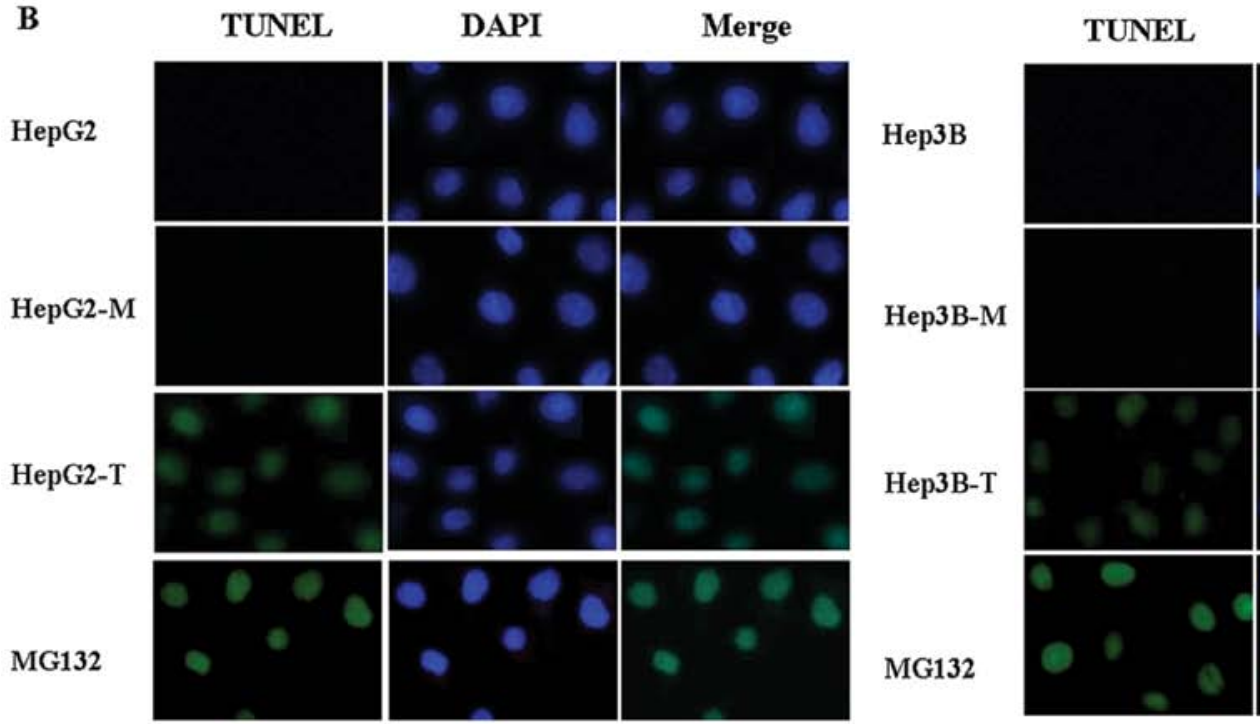

DAPI

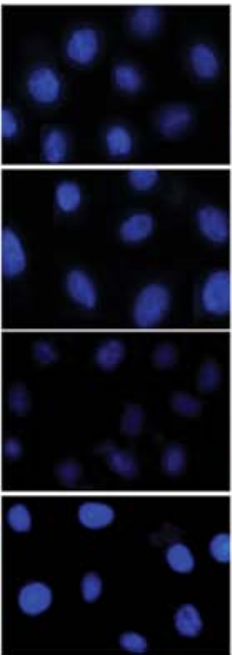

Merge

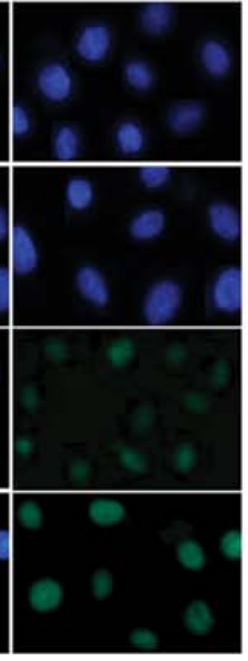

C

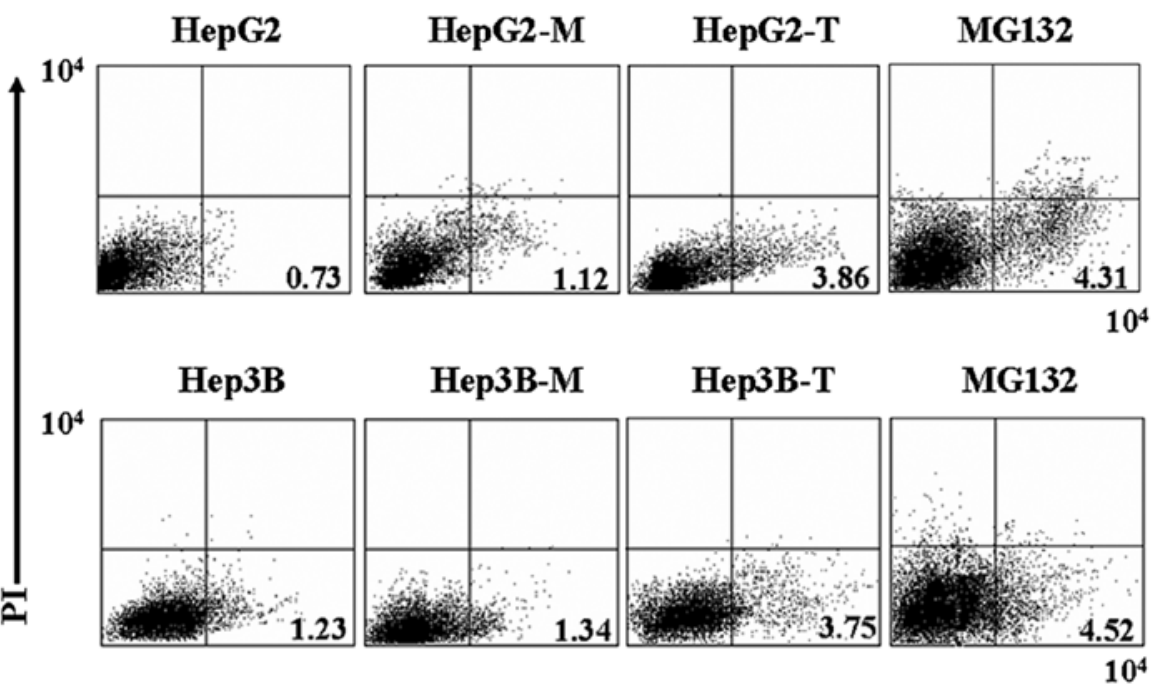

Annexin V-FITC

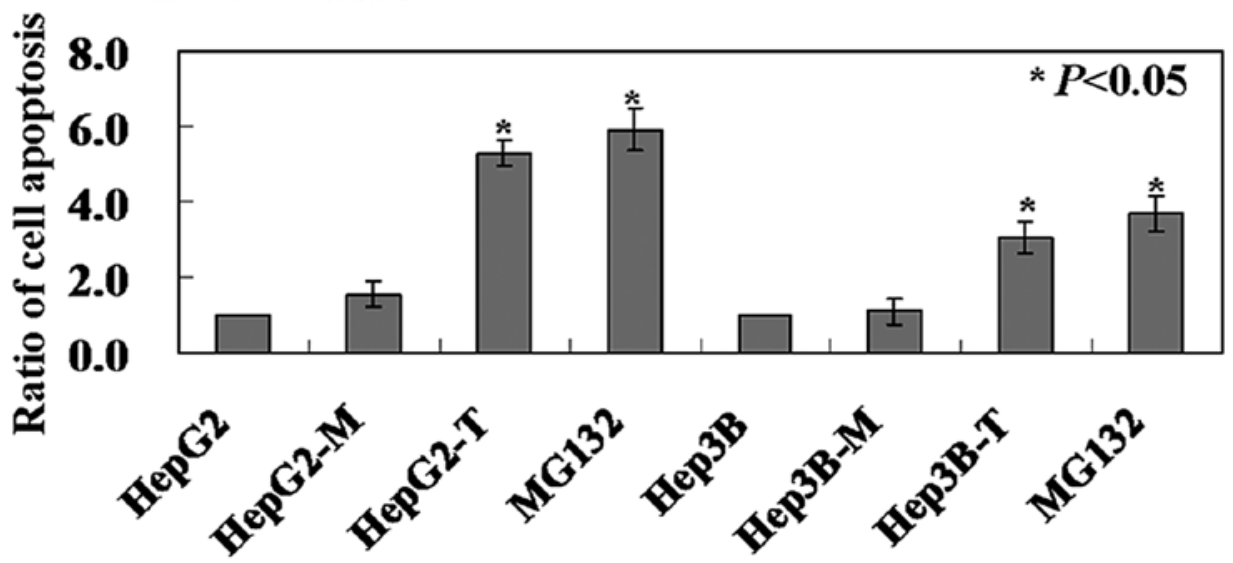


D
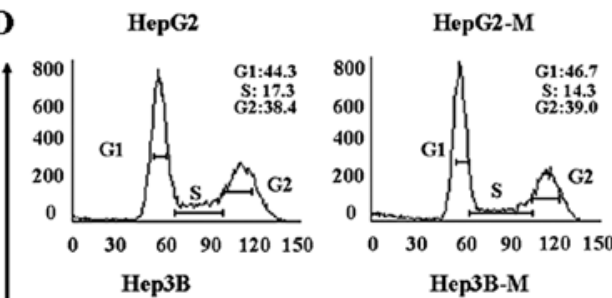

HepG2-T

MG132
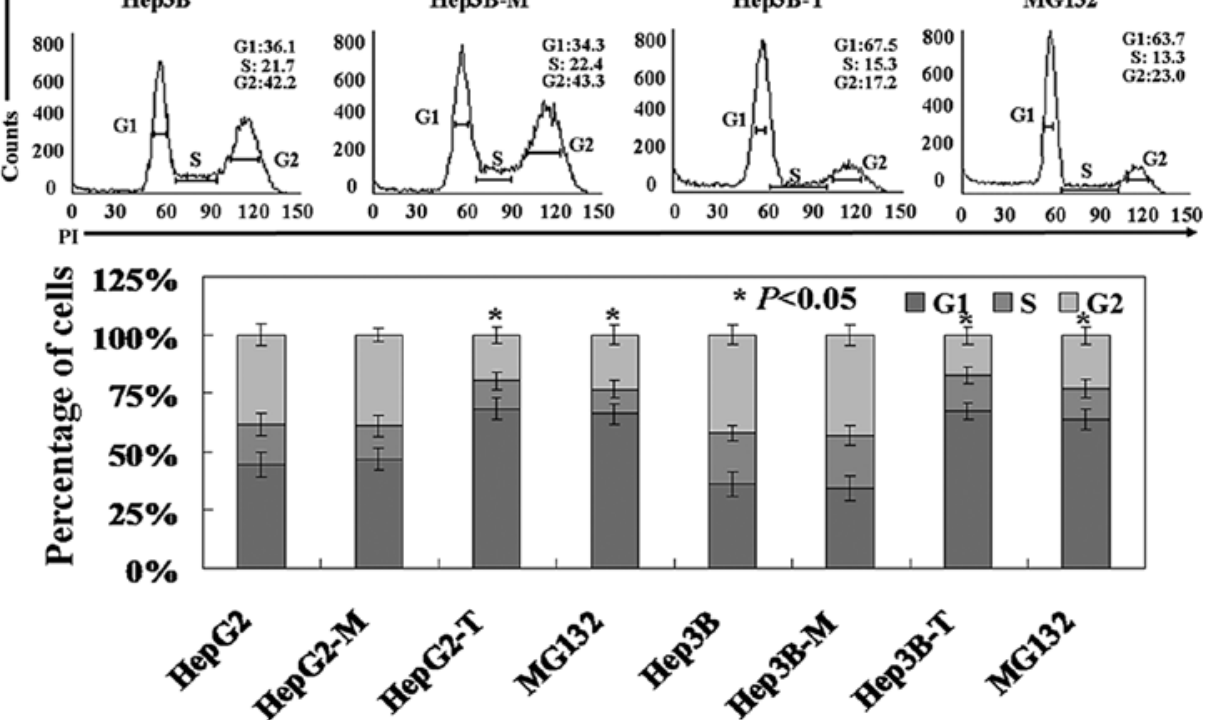

$\mathbf{E}$

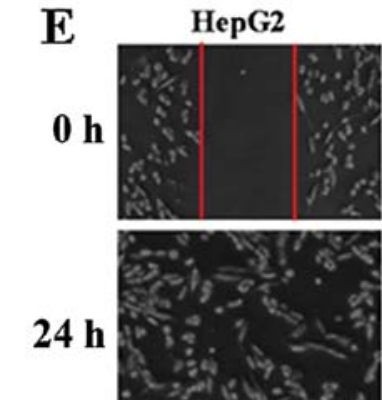

HepG2-M
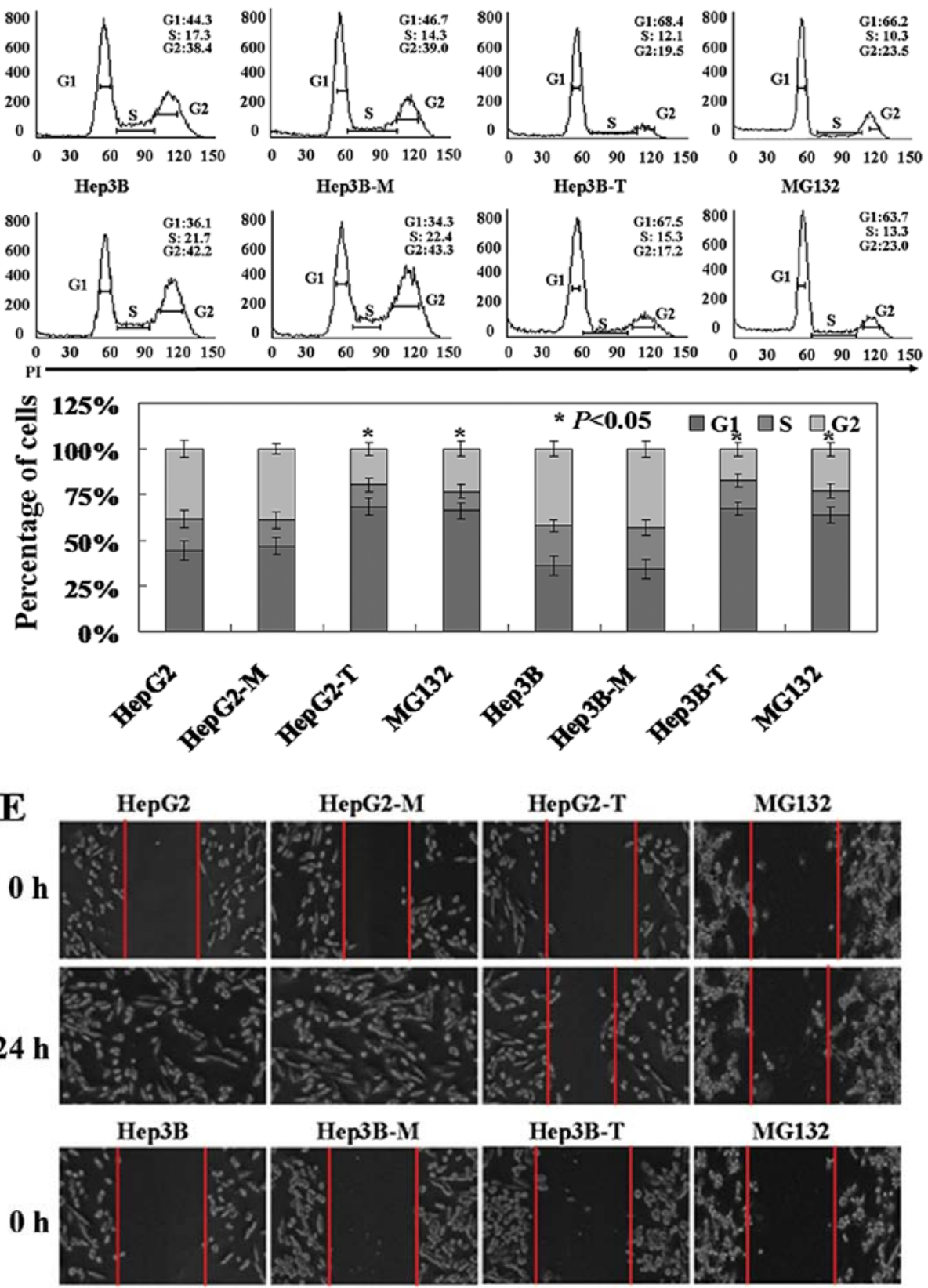

Hep3B-T

MG132

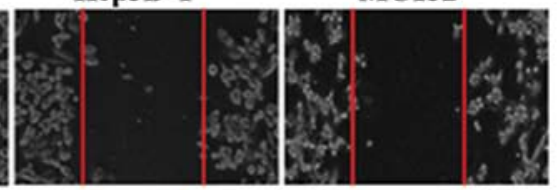

$24 \mathrm{~h}$
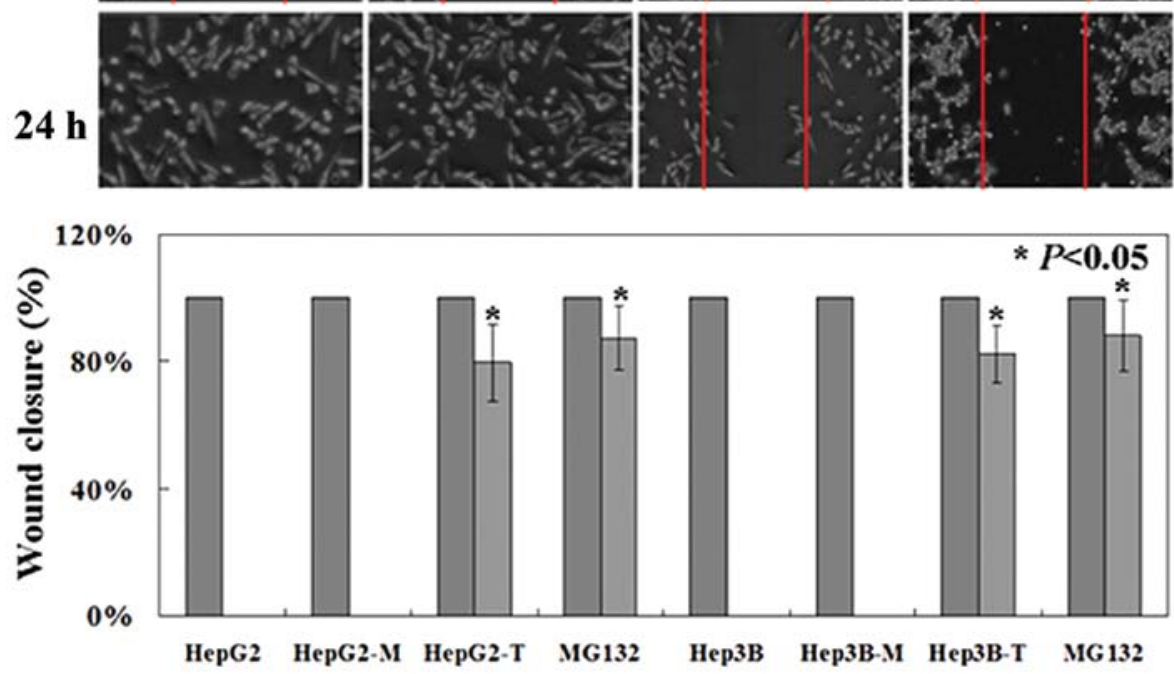

Figure 5. Continued. (D) Cells were stained with PI to analyze the cell cycle distribution of each cell type by flow cytometry. (E) Wound closure was quantified in the regions flanked by dotted lines. (F) Inhibitory effect of PDCD5 on proteasome was determined by using a microplate reader. MG132 was used as a control in each experiment. Each experiment was performed in triplicate and was repeated three times. HepG2-M, HepG2 transfected with pcDNA3.1; HepG2-T, HepG2 transfected with pcDNA3.1-PDCD5; Hep3B-M, Hep3B transfected with pcDNA3.1; Hep3B-T, Hep3B transfected with pcDNA3.1-PDCD5. 
cancer cells (Fig. 5, P<0.05). These data collectively indicate that PDCD5 suppressed HCC cells via inhibition of proteasome activity.

\section{Discussion}

Several pieces of evidence have suggested that the expression of PDCD5 protein is downregulated in some human tumors, such as breast cancer (7), gastric cancer (8) and astrocytic glioma (9). In the study of Xu et al (21), gene expression profiles of HCC and non-HCC, based on cDNA arrays or RT-PCR, reflected a number of genes with relatively high expression in non-HCC, including PDCD5. However, the clinical significance of PDCD5 expression in HCC has not been well characterized. In this study, we confirmed both PDCD5 mRNA and protein were significantly higher in normal matched tissues than that in HCC tissues. Low expression of PDCD5 in chondrosarcoma samples was significantly correlated with anatomical location and histological grade, but not with age and gender (22). The expression of PDCD5 in gliomas was correlated significantly with the pathological grade. However, we found that the PDCD5 expression was correlated statistically with HBV infection, tumor number and lymph node metastasis. The discrepancy might be attributable to clinicopathological characteristics of the subjects and grouping methods. Previous studies have documented that PDCD5 expression can be predictive for the prognosis of patients with several tumors $(8,9,22)$. We performed Kaplan-Meier analysis and confirmed PDCD5 protein was associated with the overall survival of patients with HCC. Therefore, it is implied that PDCD5 may be a favorable biomarker for the prognosis of patients with HCC. Since the sample size in this study may be considered small, further investigation of a larger patient population is necessary to confirm its clinical value and prognostic evaluation in HCC. $\alpha$-fetoprotein (AFP), is still the only serological marker presently available for routine screening in most parts of the world. High levels of AFP in fully developed hepatocarcinoma or in serum of the host are associated with more aggressive behavior and increased anaplasia $(23,24)$. In this study, the concentrations of PDCD5 and AFP in serum were lower in HCC patients than that in healthy controls and confirmed that the levels of PDCD5 and AFP were significantly positively correlated. A strong correlation between PDCD5 and IL-17 levels in serum of RA patients has been determined in a previous study (25). Wang et al (13) found decreased expression of PDCD5 in peripheral blood of breast cancer, lung cancer and gastrointestinal cancer, however, the PDCD5 levels in cancer patients are not statistically different from that of normal persons. The level of PDCD5 in serum may be used as a novel marker for HCC.

Previous studies have demonstrated that PDCD5 is an apoptosis-promoting molecule that is upregulated in cells undergoing apoptosis $(4,14,26,27)$. Likewise, blocking the activity of PDCD5 by introducing an anti-PDCD5 antibody or siRNA against PDCD5 could suppress etoposide or Baxinduced apoptosis of tumor cells $(28,29)$. In MTT assay, the proliferation ratio of transfected cells was reduced compared to untransfected ones in our study. Correspondingly, transfected cells exhibited a higher apoptotic ratio than untransfected cells by TUNEL assay and Annexin V/
PI double staining. Collectively, these results suggest that PDCD5 may have a suppressive role in HCC cells.

The mechanisms involved in PDCD5 expression are still being investigated. PDCD5 positively regulates Tip60, a transcriptional coregulator, which in turn, promotes p53 acetylation, leading to enhanced p53-dependent apoptosis (14). Another study showed that apoptotic potential of PDCD5 is linked with CK2 phosphorylation (30). It has also been shown that PDCD5 can enhance TAJ/TROY-induced paraptosis-like cell death (26). Han et al (17) found that PDCD5 mediates antitumor effects in the osteosarcoma cell line by suppressing the Ras/Raf/MEK/ERK signaling pathway. An et al (31) found that myocardial high PDCD5 overexpression results in dilated cardiomyopathy and heart failure accompanied by dramatically enhanced autophagy. Interesting, the major finding in the present study is that PDCD5 overexpression is involved in proteasome inhibition. This is supported by the fact that, PDCD5 showed the same antitumor activity as the proteosome inhibitor, MG132. The proteasome activity of transfected cells was lower than untransfected ones. Proteasome inhibition has gained interest as anticancer therapy (32). Proteasome inhibition disturbs the critical intracellular balance between proapoptotic and antiapoptotic signals shifting it towards tumor growth inhibition, apoptosis and decreased metastasis (33).

In conclusion, we have demonstrated that a decreased expression of PDCD5 correlated significantly with HBV infection, tumor number and lymph node metastasis. Although previous studies have provided insightful mechanisms of PDCD5 involved in intrinsic or extrinsic apoptotic pathways, our results showed evidence that PDCD5 is involved with proteasome inhibition. PDCD5 may serve as a novel therapeutic target for the treatment of hepatocellular cancer.

\section{Acknowledgements}

We thank Dr Miao Yu for technical assistance.

\section{References}

1. Jemal A, Bray F, Center MM, et al: Global cancer statistics. CA Cancer J Clin 6: 69-90, 2011.

2. Bosch FX, Ribes J, Diaz M, et al: Primary liver cancer: worldwide incidence and trends. Gastroenterology 127: S5-S16, 2004.

3. Perz JF, Armstrong GL, Farrington LA, et al: The contributions of hepatitis $\mathrm{B}$ virus and hepatitis $\mathrm{C}$ virus infections to cirrhosis and primary liver cancer worldwide. J Hepatol 45: 529-538, 2006.

4. Liu H, Wang Y, Zhang Y, et al: TFAR19, a novel apoptosis related gene cloned from human leukemia cell line TF21, could enhance apoptosis of some tumor cells induced by growth factor withdrawal. Biochem Biophys Res Commun 254: 203-210, 1999.

5. Spinola M, Meyer P, Kammerer S, et al: Association of the PDCD5 locus with lung cancer risk and prognosis in smokers. J Clin Oncol 24: 1672-1678, 2006.

6. Liu D, Feng Y, Cheng Y, et al: Human programmed cell death 5 protein has a helical-core and two dissociated structural regions. Biochem Biophys Res Commun 318: 391-396, 2004.

7. Hedenfalk I, Duggan D, Chen Y, et al: Gene-expression profiles in hereditary breast cancer. N Engl J Med 344: 539-548, 2001.

8. Yang Y, Zhao M, Li W, et al: Expression of programmed cell death 5 gene involves in regulation of apoptosis in gastric tumor cells. Apoptosis 11: 993-1001, 2006.

9. Li H, Wang Q, Gao F, et al: Reduced expression of PDCD5 is associated with high-grade astrocytic gliomas. Oncol Rep 20: 573-579, 2008. 
10. Ruan G, Qin Y, Chen S, et al: Abnormal expression of the programmed cell death 5 gene in acute and chronic myeloid leukemia. Leuk Res 30: 1159-1165, 2006.

11. Feng YM, Zhang YM and Jing GZ: Soluble expression in Escherichia coli, purification and characterization of a human TF-1 cell apoptosis-related protein TFAR19. Protein Expr Purif 25: 323-329, 2002.

12. Wang J, Guan Z and Ge Z: Plasma and synovial fluid programmed cell death 5 (PDCD5) levels are inversely associated with TNF- $\alpha$ and disease activity in patients with rheumatoid arthritis. Biomarkers 18: 155-159, 2013.

13. Wang Y, Wang GH and Zhang QY: Determination of PDCD5 in peripheral blood serum of cancer patients. Chin J Cancer Res 23 : 224-228, 2011.

14. Xu L, Chen Y, Song Q, et al: PDCD5 interacts with Tip60 and functions as a cooperator in acetyltransferase activity and DNA damage-induced apoptosis. Neoplasia 11: 345-354, 2009.

15. $\mathrm{Xu} \mathrm{L}, \mathrm{Hu} \mathrm{J}$, Zhao Y, et al: PDCD5 interacts with p53 and functions as a positive regulator in the p53 pathway. Apoptosis 17: $1235-1245,2012$

16. Li H, Zhang X, Song X, et al: PDCD5 promotes cisplatin-induced apoptosis of glioma cells via activating mitochondrial apoptotic pathway. Cancer Biol Ther 13: 822-830, 2012.

17. Han XR, Sun Y and Bai XZ: The antitumor role and mechanism of integrated and truncated PDCD5 proteins in osteosarcoma cells. Cell Signal 24: 1713-1721, 2012.

18. Berman DM, Karhadkar SS, Maitra A, et al: Widespread requirement for Hedgehog ligand stimulation in growth of digestive tract tumours. Nature 425: 846-851, 2003.

19. Markaryan A, Nelson EG, Tretiakova M, et al: Technical report: immunofluorescence and TUNEL staining of celloidin embedded human temporal bone tissues. Hear Res 241: 1-6, 2008.

20. Fekete MR, McBride WH and Pajonk F: Anthracyclines, proteasome activity and multi-drug-resistance. BMC Cancer 5: 114, 2005.

21. Xu XR, Huang J, Xu ZG, et al: Insight into hepatocellular carcinogenesis at transcriptome level by comparing gene expression profiles of hepatocellular carcinoma with those of corresponding non-cancer liver. Proc Natl Acad Sci USA 98: 15089-15094, 2001.
22. Chen $\mathrm{C}$, Zhou $\mathrm{H}, \mathrm{Xu} \mathrm{L}$, et al: Prognostic significance of downregulated expression of programmed cell death 5 in chondrosarcoma. J Surg Oncol 102: 838-843, 2010.

23. Dudich E, Semenkova L, Gorbatova E, et al: Growth - regulative activity of human alpha-fetoprotein for different types of tumor and normal cells. Tumour Biol 19: 30-40, 1998.

24. Wang XW and Xu B: Stimulation of tumor - cell growth by alpha-fetoprotein. Int J Cancer 75: 596-599, 1998.

25. Wang JF, Guan ZP, Zhang SL, et al: Programmed cell death 5 correlates with disease activity and interleukin-17 in serum and synovial fluid of rheumatoid arthritis patients. Chin Med J (Engl) 126: 296-299, 2013.

26. Wang Y, Li X, Wang L, et al: An alternative form of paraptosis like cell death, triggered by TAJ/TROYand enhanced by PDCD5 overexpression. J Cell Sci 117: 1525-1532, 2004.

27. Chen Y, Sun R, Han W, et al: Nuclear translocation of PDCD5 (TFAR19): an early signal for apoptosis? FEBS Lett 509: 191-196, 2001.

28. Rui M, Chen YY, Zhang YM, et al: Transfer of anti-TFAR19 monoclonal antibody into HeLa cells by in situ electroporation can inhibit the apoptosis. Life Sci 71: 1771-1778, 2002.

29. Chen LN, Wang Y, Ma DL, et al: Short interfering RNA against the PDCD5 attenuates cell apoptosis and caspase-3 activity induced by Bax overexpression. Apoptosis 11: 101-111, 2006.

30. Salvi M, Xu D, Chen Y, et al: Programmed cell death protein 5 (PDCD5) is phosphorylated by CK2 in vitro and in 293T cells. Biochem Biophys Res Commun 387: 606-610, 2009.

31. An L, Zhao X, Wu J, et al: Involvement of autophagy in cardiac remodeling in transgenic mice with cardiac specific over-expression of human programmed cell death 5. PLoS One 7: e30097, 2012.

32. Adams J: The development of proteasome inhibitors as anticancer drugs. Cancer Cell 5: 417-421, 2004

33. Wu WK, Cho CH, Lee CW, et al: Proteasome inhibition: a new therapeutic strategy to cancer treatment. Cancer Lett 293: 15-22, 2010. 